\author{
Case Reports
}

\title{
Melancholic features related to rimonabant
}

\author{
Bernardo de Mattos Viana, M.D., ${ }^{\mathrm{a}, *}$, Hugo Alejandro Cano Prais, M.D., M.Sc. ${ }^{\mathrm{b}}$, \\ Maurício Viotti Daker, M.D., Ph.D. ${ }^{\mathrm{c}, \mathrm{d}}$ \\ ${ }^{a}$ Psychiatry Service, Hospital das Clínicas (HC-UFMG), Federal University of Minas Gerais (UFMG), Belo Horizonte (MG), Brazil \\ ${ }^{\mathrm{b}}$ Departament of Medical Sciences, Federal University of Ouro Preto (UFOP), Ouro Preto (MG), Brazil \\ ${ }^{\mathrm{c}}$ Psychiatry Service, Hospital das Clínicas (HC-UFMG), Federal University of Minas Gerais (UFMG), Belo Horizonte (MG), Brazil \\ ${ }^{\mathrm{d}}$ Department of Mental Health, School of Medicine, Federal University of Minas Gerais (UFMG), Belo Horizonte (MG), Brazil \\ Received 8 October 2008; accepted 30 December 2008
}

\begin{abstract}
Introduction: Obesity is currently considered a global epidemic and its prevention and treatment is a major public health concern, demanding treatment which may combine a sociocultural approach, lifestyle modification, nutritional, pharmacological or surgical strategies. Rimonabant, an endocannabinoid antagonist, has been proposed as an agent for an average weight loss of $4 \mathrm{~kg}$. However, the development of anxiety and depressive symptoms can be major side effects.

Case Report: A 27-year-old businessman, after using rimonabant (20 mg/day) for 1 month for weight loss, developed a major depressive episode with melancholic features, which remitted after the interruption of rimonabant.

Discussion: To our knowledge, a major depressive episode with melancholic or atypical features specifier has not been described since the initiation of rimonabant pharmacological trials. The Hospital Anxiety and Depression Scale, used in the rimonabant trials, assesses several key points of depressive patients. However, it neglects the somatic symptoms that correspond to the additional criterion for both of the mentioned features as well as suicidal ideation. The severity of an episode could also be underestimated depending on the assessment tool or on the clinical interview.

Conclusion: There may be an underestimation of depressive melancholic and atypical side effects related to Rimonabant use, due to the lack of consistent assessment with the appropriate screening tools. Pharmacological strategies should be adjunctive for obesity treatment when there is a failure in the lifestyle and nutritional modification strategies. Moreover, deeper global sociocultural changes should be made in the treatment and control of the global obesity epidemic.
\end{abstract}

(C) 2009 Elsevier Inc. All rights reserved.

Keywords: Rimonabant; Depression; Melancholic features; Psychiatric Status Rating Scales; Obesity

\section{Introduction}

Obesity has long been associated with sedentary lifestyles and high-fat/energy-dense diets, and it is currently considered a global epidemic [1]. The prevention and treatment of obesity and its serious comorbidities is a major public health challenge, generally demanding a combination of strategies: a sociocultural approach, lifestyle modification, nutrition, pharmacology and/or surgery $[2,3]$.

* Corresponding author. Serviço de Psiquiatria do Hospital das Clínicas da Universidade Federal de Minas Gerais. Av. Prof. Alfredo Balena 110, Santa Efigênia. Belo Horizonte, MG, Brasil. CEP: 30130-100. Tel.: +55 31 3409 9458; fax: +55 3134099458 .

E-mail address: bernardomviana@yahoo.com (B. de Mattos Viana).
Indications for pharmacological treatment include patients with a body-mass index (BMI) of $27 \cdot 0-29 \cdot 9 \mathrm{~kg} /$ $\mathrm{m}^{2}$ with a major obesity comorbidity or a BMI of $30 \mathrm{~kg} / \mathrm{m}^{2}$ and over [4]. The three main targets are the psychological experience and behavioral expression of appetite, the peripheral physiology or metabolism and the modulation of the central nervous system neural pathways [5]. The use of antiobesity agents in general presents good response in weight loss, but the Food and Drug Administration (FDA) currently licenses only orlistat and sibutramine for long-term use [6]. Orlistat's main adverse effects is fatty and oily stool, fecal urgency and oily spotting, while sibutramine's side effects are insomnia, nausea, dry mouth, constipation and slight increases in blood pressure and pulse rate [6]. Rimonabant is marketed as another option, but some studies 
point to a relevant risk for developing anxiety and depressive symptoms [7-11].

\section{Case report}

Mr. A. came to his first psychiatric consultation at the suggestion of his urologist due to complaints about precocious ejaculation. There were no physical problems associated with this symptom, and all the laboratory workup, including thyroid screening, was unremarkable. There were no depressive symptoms, just anticipatory anxiety related to his sexual complaints. He mentioned no sleep or appetite disturbances and enthusiastically described his work and his hobby, dog raising. There was no family history of major depression or other psychiatric disorders. The patient was treated with clomipramine $10 \mathrm{mg} /$ day, and insightoriented psychotherapy was indicated.

One month later, he presented a good pharmacological response with no anxiety symptoms. He was euthimic without sleep or appetite disturbances. He described his life as happy and mentioned his motivation to get married. He received a recommendation to extend the use of clomipramine for 2 more months while continuing psychotherapy.

After 2 months, the patient requested a new consultation. He reported feeling unmotivated to work with a remarkable loss of interest in sex and other pleasures like going out with friends during the past 2 weeks. He did not feel any better even after his dog gave birth, showing a lack of mood reactivity. He complained about early morning awakening and a marked psychomotor agitation with a consequent perception of worsening of symptoms and mood at the beginning of the day. He denied feelings of hopelessness, excessive guilt, suicidal ideation or stressful life events.

He was evidently thinner than at the previous consultation and informed the physician that he had been prescribed rimonabant for 1 month with subsequent weight loss of $4 \mathrm{~kg}$, despite a previous BMI of 23.3. His endocrinologist had prescribed it in order for him to get "in shape" without dietary restrictions or physical activities. The depressive symptoms began 15 days after the start of rimonabant $20 \mathrm{mg} /$ day and he was no longer using clomipramine.

He was advised to interrupt rimonabant, and 1 month later, he experienced improvement in abulic and anhedonic symptoms, sleep and psychomotor agitation.

\section{Discussion}

It is suggested that increased activity of the endocannabinoid system might contribute to the development of obesity and metabolic syndromes and sustained by the leptin resistance [7]. Rimonabant is an endocannabinoid, cannabinoid-1 receptor antagonist that reduces waist circumference; optimizes high-density lipoprotein cholesterol and triglyceride concentrations and produces an average of $4.7 \mathrm{~kg}$ of weight loss, after 1 year of $20-\mathrm{mg} /$ day use $[6,8,9]$.
In a recent meta-analysis [9] rimonabant was associated with 2.5 times the chance of discontinuing the treatment due to depressive symptoms and a 3.0 times chance due to anxiety, compared to a placebo. The FDA briefing document reported a relative risk of $1.9(\mathrm{CI}, 1.5,2.3)$ for psychiatric adverse event compared to placebo, as well as an increased risk (odds ratio, 1.9) of suicidality (CI, 1.1, 3.1) $[10,11]$ Nine percent of the rimonabant $20 \mathrm{mg}$-treated subjects reported symptoms of depression vs. $5 \%$ of placebo users [11]. It is important to note that a history of major depressive disorder was an exclusion criterion for patients in some studies [9].

To our knowledge, this is the first report of a major depressive episode with melancholic features since the initiation of rimonabant pharmacological trials. The depression screening tool used in most rimonabant trials is the Hospital Anxiety and Depression Scale (HADS), a self-rating scale, which is interesting because it has only cognitive and emotional symptoms, thereby excluding the effect of the somatic symptoms that could be related to disorders like cancer, obesity and obstructive sleep apnea $[12,13]$. On the other hand, it does not measure the onset of new depressive somatic symptoms, especially melancholic or atypical features secondary to the use of the medication. Another key point in depression assessment is suicidal ideation or attempt presenting an eminent risk for the patient, which is also not screened by the HADS. It is indeed a valid and important scale [14]; however, there is also a concern that the observer-rating scales, per se, are more valid than selfrating [15]. Nevertheless, even the Hamilton Depression Rating Scale $\left(H A M-D_{17}\right)$, still considered the gold standard for outcome measurement in trials with antidepressive medication, is questioned because of the lack of incorporation of contemporary definitions of depression [16]. In addition, HAM-D ${ }_{17}$ does not assess some of the criterion of melancholic, especially atypical features, as well as the Montgomery-Åsberg Depression Rating Scale and the Beck Depression Inventory [17]. Moreover, severity and specific features of an episode cannot be screened solely by the assessment tool.

According to the Diagnostic and Statistical Manual of Mental Disorders, Fourth Edition, Text Revision, our patient fulfilled the following criteria for a major depressive episode with a melancholic features specifier: A1 (loss of pleasure in all or most activities), A2 (lack of reactivity to normally pleasurable stimuli), B2 (depression regularly worse in the morning), B3 (early morning awakening) and B4 (marked psychomotor retardation or agitation). It is indeed hard to evaluate the correlation of criterion B5 (significant anorexia or weight loss) with the melancholic feature of the depressive episode, as it may be directly associated with the use of rimonabant. Nonetheless, the patient lost $4 \mathrm{~kg}$ in only 1 month, which could be related to the development of the depressive episode and, therefore, significantly increased it.

\section{Conclusion}

There may be an underestimation of depressive melancholic and atypical side effects related to rimonabant use, 
due to the lack of consistent assessment with the appropriate screening tools. However, there is limited evidence based on case reports, this being the first to report melancholic features. Conversely, studies of rimonabant have shown substantial reduction of some of the cardiometabolic risk factors that are not generally associated with sibutramine or orlistat [6]. Thus, more data on risks and benefits of this drug is still needed.

Treatment of the obesity epidemic is a hard task. Pharmacological treatment strategies should be adjunctive and used when there is a failure of the lifestyle and nutritional modification strategies. The inability of pharmacological treatment alone to reverse obesity trend shows how deeper changes need to be proposed by public health providers and health professionals. Influences related to modern sociocultural issues may play the central role in dealing with this epidemic. Depression rates also show an upward trend and may also be related to sociocultural changes [18].

Physical activities should not be restricted to fitness centers and questions about transportation and modern labor duties should also be included. The previous cultural dietary regionalism was changed to a globalized high-fat/energydense fast food that is proposed to be in accordance with busy time schedules. This global change in diet is now likely to increase the rates of obesity in the majority of societies, with the inevitable outcome of increased weight concerns and eating psychopathology [19].

These are also some of the questions that demand further investigation on obesity epidemiology and treatment.

\section{References}

[1] WHO. Obesity: preventing and managing the global epidemic. WHO Technical Report Series number 894. Geneva: WHO; 2000.

[2] Haslam DW, James WP. Obesity. Lancet 2005;366:1197-209.

[3] Swinburn B, Egger G. Preventive strategies against weight gain and obesity. Obes Rev 2002;3:289-301.
[4] National Institutes of Health/National Heart, Lung, and Blood Institute Obesity Education Initiative. Clinical guidelines on the identification, evaluation, and treatment of overweight and obesity in adults: the evidence report. Bethesda (MD): US Department of Health and Human Services; 1998.

[5] Halford JC. Pharmacology of appetite suppression: implication for the treatment of obesity. Curr Drug Targets 2001;2:353-70.

[6] Padwal RS, Majumdar SR. Drug treatments for obesity: orlistat, sibutramine, and rimonabant. Lancet 2007;369:71-7.

[7] Di Marzo V, Matias I. Endocannabinoid control of food intake and energy balance. Nat Neurosci 2005;8:585-9.

[8] Curioni C, André C. Rimonabant for overweight or obesity. Cochrane Database Syst Rev 2006;18:CD006162.

[9] Christensen R, Kristensen PK, Bartels EM, Bliddal H, Astrup A. Efficacy and safety of the weight-loss drug rimonabant: a metaanalysis of randomised trials. Lancet 2007;370:1706-13.

[10] Mitchell PB, Morris MJ. Depression and anxiety with rimonabant. Lancet 2007;370:1671-2.

[11] US Food and Drug Administration Advisory Committee. Rimonabant Briefing Document: NDA 21-888. Zimulti (rimonabant) Tablets, $20 \mathrm{mg}$. Rockville (MD): FDA; 2007. http://www.fda.gov/ohrms/dockets/ac/07/ briefing/2007-4306b1-fda-backgrounder.pdf. (assessed Aug 10, 2008).

[12] Zigmond AS, Snaith RP. The Hospital Anxiety and Depression Scale. Acta Psychiatr Scand 1983;67:361-70.

[13] Razavi D, Delvaux N, Farvacques C, Robaye E. Screening for adjustment disorders and major depressive disorders in cancer inpatients. Br J Psychiatry 1990;156:79-83.

[14] Bjelland I, Dahl AA, Haug TT, Neckelmann D. The validity of the Hospital Anxiety and Depression Scale. An updated literature review. J Psychosom Res 2002;52:69-77.

[15] Smolka M, Stieglitz RD. On the validity of the Bech-Rafaelsen Melancholia Scale (BRMS). J Affect Disord 1999;54:119-28.

[16] Bagby RM, Ryder AG, Schuller DR, Marshall MB. The Hamilton Depression Rating Scale: has the gold standard become a lead weight? Am J Psychiatry 2004;161:2163-77.

[17] Demyttenaere K, De Fruyt J. Getting what you ask for: on the selectivity of depression rating scales. Psychother Psychosom 2003;72:61-70.

[18] Compton WM, Conway KP, Stinson FS, Grant BF. Changes in the prevalence of major depression and comorbid substance use disorders in the United States between 1991-1992 and 2001-2002. Am J Psychiatry 2006;163:2141-7.

[19] Nasser M. Culture and eating disorders. In: Bhugra D, Bhui K, editors. Textbook of Cultural Psychiatry. New York: Cambridge University Press; 2007. p. 292-300. 